\title{
GENERALIZED WEIGHTED OSTROWSKI AND OSTROWSKI-GRÜSS TYPE INEQUALITIES ON TIME SCALES VIA A PARAMETER FUNCTION
}

\author{
Seth Kermausuor, Eze R. Nwaeze and Delfim F. M. Torres
}

Abstract. We prove generalized weighted Ostrowski and Ostrowski-Grüss type inequalities on time scales via a parameter function. In particular, our result extends a result of Dragomir and Barnett. Furthermore, we apply our results to the continuous, discrete, and quantum cases, to obtain some interesting new inequalities.

Mathematics subject classification (2010): 26D10, 26D15, 26E70. scales.

Keywords and phrases: Ostrowski's inequality, Ostrowski-Grüss inequality, parameter function, time

\section{REFERENCES}

[1] Ravi Agarwal, Donal O'Regan and Samir Saker, Dynamic inequalities on time scales, Springer, Cham, 2014.

[2] R. Agarwal, M. Bohner And A. Peterson, Inequalities on time scales: a survey, Math. Inequal. Appl. 4 (2001), no. 4, 535-557, DOI 10.7153/mia-04-48.

[3] George A. Anastassiou, Frontiers in time scales and inequalities, Series on Concrete and Applicable Mathematics, vol. 17, World Scientific Publishing Co. Pte. Ltd., Hackensack, NJ, 2016.

[4] Khalid Mahmood Awan, Josip PeČarić and Mihaela Ribičić Penava, Companion inequalities to Ostrowski-Grüss type inequality and applications, Turkish J. Math. 39 (2015), no. 2, 228-234, DOI 10.3906/mat-1404-27.

[5] Josipa Barić, Rabia Bibi, Martin Bohner, Ammara Nosheen and Josip Pečarić, Jensen inequalities on time scales, Monographs in Inequalities, vol. 9, Element, Zagreb, 2015, Theory and applications.

[6] Martin Bohner and Thomas Matthews, The Grüss inequality on time scales, Commun. Math. Anal. 3 (2007), no. 1, 1-8.

[7] Martin Bohner and Thomas Matthews, Ostrowski inequalities on time scales, JIPAM. J. Inequal. Pure Appl. Math. 9 (2008), no. 1, Article 6, 8.

[8] Martin Bohner and Allan Peterson, Dynamic equations on time scales, Birkhäuser Boston, Inc., Boston, MA, 2001, An introduction with applications.

[9] Martin Bohner And Allan Peterson, Advances in dynamic equations on time scales, Birkhäuser Boston, Inc., Boston, MA, 2003.

[10] S. S. DRAgomir And N. S. Barnett, An Ostrowski type inequality for mappings whose second derivatives are bounded and applications, J. Indian Math. Soc. (N.S.) 66 (1999), no. 1-4, 237-245.

[11] STEFAN HILGER, Analysis on measure chains - a unified approach to continuous and discrete calculus, Results Math. 18 (1990), no. 1-2, 18-56, DOI 10.1007/BF03323153.

[12] Victor Kac and Pokman Cheung, Quantum calculus, Universitext, Springer-Verlag, New York, 2002.

[13] Wenjun Liu, Some weighted integral inequalities with a parameter and applications, Acta Appl. Math. 109 (2010), no. 2, 389-400, DOI 10.1007/s10440-008-9323-2.

[14] Wenjun Liu, Quõc Anh NGÔ And Wenbing Chen, Ostrowski type inequalities on time scales for double integrals, Acta Appl. Math. 110 (2010), no. 1, 477-497, DOI 10.1007/s10440-009-9456-y.

[15] Wenjun Liu, Quõc AnH NGô And Wenbing Chen, On new Ostrowski type inequalities for double integrals on time scales, Dynam. Systems Appl. 19 (2010), no. 1, 189-198. 
[16] Wenjun Liu, Adnan Tuna And Yong Jiang, On weighted Ostrowski type, trapezoid type, Grüss type and Ostrowski-Grüss like inequalities on time scales, Appl. Anal. 93 (2014), no. 3, 551-571, DOI 10.1080/00036811.2013.786045.

[17] Wenjun Liu, Adnan Tuna And Yong Jiang, New weighted Ostrowski and Ostrowski-Grüss type inequalities on time scales, An. Ştiinţ. Univ. Al. I. Cuza Iaşi. Mat. (N.S.) 60 (2014), no. 1, 57-76, DOI 10.2478/aicu-2013-0002.

[18] Dorota Mozyrska, Ewa PawŁuszewicz and Delfim F. M. Torres, Inequalities and majorisations for the Riemann-Stieltjes integral on time scales, Math. Inequal. Appl. 14 (2011), no. 2, 281-293, DOI 10.7153/mia-14-23.

[19] EzE R. NwAEZE, A new weighted Ostrowski type inequality on arbitrary time scale, J. King Saud Univ. Sci. 29 (2017), no. 2, 230-234, DOI 10.1016/j.jksus.2016.09.006.

[20] Umut Mutlu ÖZKan And HÜSEYIn Yildirim, Grüss type inequalities for double integrals on time scales, Comput. Math. Appl. 57 (2009), no. 3, 436-444, DOI 10.1016/j.camwa.2008.11.006.

[21] Umut MutLu ÖZKAN AND HÜSEYIN YILDIRIM, Ostrowski type inequality for double integrals on time scales, Acta Appl. Math. 110 (2010), no. 1, 283-288, DOI 10.1007/s10440-008-9407-z.

[22] Gaopeng Xu and Zhong Bo Fang, A new Ostrowski type inequality on time scales, J. Math. Inequal. 10 (2016), no. 3, 751-760. 